Published in final edited form as:

Obesity (Silver Spring). 2019 March ; 27(3): 420-426. doi:10.1002/oby.22389.

\title{
Objective versus Self-Reported Energy Intake Changes During Low-Carbohydrate and Low-Fat Diets
}

\author{
Juen Guo ${ }^{1}$, Jennifer L. Robinson ${ }^{2}$, Christopher Gardner ${ }^{2}$, and Kevin D. Hall ${ }^{1, \dagger}$ \\ ${ }^{1}$ National Institute of Diabetes and Digestive and Kidney Diseases \\ ${ }^{2}$ Stanford University
}

\begin{abstract}
Objective: To compare self-reported with objective measurements of energy intake changes ( $\Delta \mathrm{EI}$ ) during a 1-year weight loss intervention with subjects randomized to low-carbohydrate versus low-fat diets.
\end{abstract}

Methods: We used repeated body weight measurements as inputs to an objective mathematical model to calculate $\Delta \mathrm{EI}_{\text {Model }}$ to compare with self-reported energy intake changes assessed by repeated 24 -hr recalls $\left(\Delta \mathrm{EI}_{\text {Recall }}\right)$.

Results: $\Delta \mathrm{EI}_{\text {Recall }}$ indicated a relatively persistent state of calorie restriction of $\sim 500-600 \mathrm{kcal} / \mathrm{d}$ at 3,6, and 12 months with no significant differences between the diets. $\Delta \mathrm{EI}_{\text {Model }}$ demonstrated large early decreases in calorie intake $>800 \mathrm{kcal} / \mathrm{d}$ followed by an exponential return to $\sim 100$ $\mathrm{kcal} / \mathrm{d}$ below baseline at the end of the year. Accounting for self-reported physical activities did not materially affect the results. Discrepancies between $\Delta \mathrm{EI}_{\text {Model }}$ and $\Delta \mathrm{EI}_{\text {Recall }}$ became progressively greater over time. The low-carbohydrate diet resulted in $\Delta \mathrm{EI}_{\text {Model }}$ that was $162 \pm 53$ $\mathrm{kcal} / \mathrm{d}$ lower than the low-fat diet over the first 3 months $(\mathrm{p}=0.002)$, but no significant diet differences were found thereafter.

Conclusions: Self-reported $\Delta \mathrm{EI}$ measurements were inaccurate. Model-based calculations of $\Delta \mathrm{EI}$ found that instructions to follow the low-carbohydrate diet resulted in greater calorie restriction than the low-fat diet in the early phases of the intervention, but these diet differences were not sustained.

\section{Keywords}

Energy intake; diet composition

\section{Introduction}

Diet assessment instruments that rely on self-report, such as $24-\mathrm{hr}$ recall, are known to substantially underestimate energy intake (1). However, repeated self-reported

Users may view, print, copy, and download text and data-mine the content in such documents, for the purposes of academic research, subject always to the full Conditions of use:http://www.nature.com/authors/editorial_policies/license.html\#terms

${ }^{\dagger}$ To whom correspondence should be addressed: Kevin D. Hall, Ph.D., National Institute of Diabetes \& Digestive \& Kidney Diseases, 12A South Drive, Room 4007, Bethesda, MD 20892, kevinh@ @iddk.nih.gov.

Conflict of interest disclosure statement: None of the authors have conflicts of interest 
measurements could possibly track changes in energy intake accurately if the measurement bias is roughly constant for each subject. For example, if a person habitually eats a weight maintenance diet of $2500 \mathrm{kcal} / \mathrm{d}$ then their 24-hr recall might under-report eating only 1900 $\mathrm{kcal} / \mathrm{d}$. If they consistently underestimated their energy intake, then after starting a weight loss diet program they might report eating $1400 \mathrm{kcal} / \mathrm{d}$ whereas they actually consumed $2000 \mathrm{kcal} / \mathrm{d}$. Their reported absolute energy intake would still be $600 \mathrm{kcal} / \mathrm{d}$ too low, but the self-reported change in energy intake of $500 \mathrm{kcal} / \mathrm{d}$ would be accurate. It is presently unknown whether people can accurately report changes in energy intake during a weight loss intervention.

We recently validated an objective mathematical method for calculating energy intake changes over time using only information about age, sex, height, and repeated measurements of body weight (2). Here, we applied this method to data from the Diet Intervention Examining The Factors Interacting with Treatment Success (DIETFIITS) randomized weight loss trial (3) and compared the model-calculated energy intake changes with selfreported values determined by repeated $24 \mathrm{hr}$ recalls.

\section{Methods}

As previously described $(3,4)$, participants were randomized to the low-carbohydrate or low-fat diet groups and were instructed reduce intake of total fat or digestible carbohydrates to $20 \mathrm{~g} / \mathrm{d}$ during the first 8 weeks and then slowly add fats or carbohydrates back to their diets in increments of 5 to $15 \mathrm{~g} / \mathrm{d}$ per week until they reached the lowest level of intake they believed could be maintained indefinitely. No instructions were provided regarding calorie restriction.

As previously described $(3,4)$, self-reported dietary intake was assessed using 3 unannounced 24-hour multiple-pass recall interviews ( 2 on weekdays and 1 on a weekend day) administered before the intervention and again after approximately 3, 6 and 12 months. Data were collected using Nutrition Data System for Research (NDSR), a computer-based software application developed at the University of Minnesota Nutrition Coordinating Center (http://www.ncc.umn.edu/products/). Dietary recalls were collected in a standardized fashion using a multiple-pass interview approach consisting of five steps to ensure completeness and accuracy. Throughout the recall, the NDSR software searched for foods and brand name products by name and prompted the data collectors with requests for additional detailed information. In addition, the interviewers entered recipes or ingredients for homemade, restaurant, and other items not included in the software. All data collectors were trained by NDSR certified lead staff and were blinded to the assigned diets. The lead dietary assessment nutritionist conducted a quality check for each cohort after data collection at each study collection point. This involved an in-depth review of both individual and composite reports for completeness and errors.

Body weight was measured by digital scale at the Stanford Clinical Translational Research Unit. Self-reported body weight was also recorded when subjects participated in the 22 instructional sessions over the course of the year. We used data from 414 subjects in the DIETFITS study (209 subjects randomized to the low-carbohydrate diet and 205 subjects 
randomized to the low-fat diet) with complete body weight data at all clinic visits. Of the subjects with complete clinic weight data, only one was missing baseline self-reported energy intake and 3,11, and 13 were missing self-reported energy intake at 3, 6, and 12 months, respectively.

As previously described (2), we used a linearized mathematical model of body weight dynamics that was solved for the change in energy intake averaged over each time interval $i$ as compared to a weight-maintaining baseline diet, $\Delta \mathrm{EI}_{\text {Model }}$, as a function of body weight and its rate of change as follows:

$$
\Delta E I_{i}=\rho \frac{d B W_{i}}{d t}+\varepsilon_{i}\left(\overline{B W}_{i}-B W_{0}\right)+\frac{\Delta \delta_{i}}{1-\beta} B W_{0}
$$

where $\rho$ is an effective energy density associated with the $B W$ change:

$$
\rho=\frac{\eta_{F M}+\rho_{F M}+\alpha \eta_{F F M}+\alpha \rho_{F F M}}{(1-\beta)(1+\alpha)}
$$

and $\varepsilon_{i}$ is a parameter that defines how energy expenditure depends on $B W$ :

$$
\varepsilon_{i}=\frac{1}{(1-\beta)}\left[\frac{\gamma_{F}+\alpha \gamma}{(1+\alpha)}+\delta_{0}+\Delta \delta_{i}\right]
$$

The parameters $\gamma_{F F M}$ and $\gamma_{F M}$ are the regression coefficients relating resting metabolic rate to fat-free mass (FFM) and fat mass (FM), respectively. Parameters $\rho_{F M}$ and $\rho_{F F M}$ are the energy densities associated with changes in FM and FFM, respectively. Physical activity energy expenditure is proportional to body weight, where $\delta_{O}$ represents the baseline level of physical activity and $\Delta \delta_{i}$ is the change in physical activity from baseline over each time period. The parameter $\beta$ accounts for the adaptation of energy expenditure during a diet perturbation, $\Delta E I$. Parameters $\eta_{F M}$ and $\eta_{F F M}$ account for the biochemical cost of tissue deposition and turnover assuming that the change of FFM is primarily accounted for by body protein and its associated water. The parameter $a$ represents the relationship between changes of fat-free and fat mass: $a \equiv d F F M / d F M=C F M$ where $\mathrm{C}=10.4 \mathrm{~kg}$ is the Forbes parameter. For modest weight changes, $a$ can be considered to be approximately constant with $F M$ fixed at its initial value $\mathrm{FM}_{0}$. The larger the initial fat mass, $\mathrm{FM}_{0}$, the smaller the parameter $a$.

The model parameters are listed in Supplementary Table 1 and we used the initial age, sex, and height to calculate the parameter $a$ for each subject. For the main analysis, we assumed that the baseline physical activity parameter was $\delta_{O}=10 \mathrm{kcal} / \mathrm{kg} / \mathrm{d}$ corresponding to an initial free-living physical activity level (PAL) 1.6. Therefore, the average linearized model parameters were (mean $\pm \mathrm{SE}$ ) $\rho=10036 \pm 21 \mathrm{kcal} / \mathrm{kg}$ and $\varepsilon=23 \pm 0.05 \mathrm{kcal} / \mathrm{kg} / \mathrm{d}$ assuming no physical activity changes (i.e. $\Delta \delta_{i}=0$ ). We also conducted an analysis of the subset of subjects $(\mathrm{N}=338)$ with self-reported physical activities at baseline and 12 months $(5)$ to define individual values for $\delta_{0}$ and $\Delta \delta_{i}$ where we linearly interpolated between the times 
when $\Delta \delta_{i}$ was measured. The mean values were $\delta_{0}=8.8 \pm 0.1 \mathrm{kcal} / \mathrm{kg} / \mathrm{d}, \Delta \delta_{i}=0.4 \pm 0.1$ $\mathrm{kcal} / \mathrm{kg} / \mathrm{d}$ at both $i=3$ and 6 months, and $\Delta \delta_{i}=0.5 \pm 0.1 \mathrm{kcal} / \mathrm{kg} / \mathrm{d}$ at $i=12$ months.

The change of mean body weight versus baseline over each interval, $\left(\overline{B W}_{i}-B W_{0}\right)$, and the moving average of the measured body weight time course was used to calculate the rate of change of body weight over each interval, $d B W_{i} / d t$. The interval length was $t=(N-1) * T$, where $N$ was the number of body weight measurements per interval and $T$ was the number of days between measurements. When clinic weights were used, $\mathrm{N}=2$ for all periods and $\mathrm{T}=90$ days for the first and second 3-month periods and $\mathrm{T}=180$ days for the final 6 months. When self-reported weights were used, we specified the interval lengths of $t=30$ days, $t=60$ days, and $\mathrm{t}=90$ days to calculate the average $\Delta \mathrm{EI}_{\text {Model }}$ and the values for $\mathrm{N}$ and $\mathrm{T}$ were calculated using the available data on each subject in the corresponding time interval. In the figures, $\Delta \mathrm{EI}_{\text {Model }}$ values were plotted at the midpoint time of each averaging interval.

Exponential time courses were fit to $\Delta \mathrm{EI}_{\text {Model }}$ values using Berkeley Madonna software (version 8.3) with equal weight given to the values determined by clinic and self-reported weights since they all appeared to lie on the same curve. Statistical analysis was performed using SAS (version 9.4) and a paired, two-sided t-test with significance declared at the $\mathrm{p}<0.05$ threshold. The data are reported as mean \pm SE.

\section{Results}

Figure 1A shows the mean weight changes measured at the clinic visits as well as those recorded at group counseling sessions where participants reported their weights as somewhat lower than could be documented in the clinic. Figure 1B illustrates the model-based measurements as well as the self-reported measurements of energy intake change. After 3 months of the intervention, $\Delta \mathrm{EI}_{\text {Recall }}=-641 \pm 31 \mathrm{kcal} / \mathrm{d}$ which was significantly lower than $\Delta \mathrm{EI}_{\text {Recall }}=-547 \pm 32 \mathrm{kcal} / \mathrm{d}$ at 6 months $(\mathrm{p}<0.0001)$. At $12 \mathrm{months}, \Delta \mathrm{EI}_{\text {Recall }}=-500 \pm 31 \mathrm{kcal} / \mathrm{d}$ and was similar to the value at 6 months $(\mathrm{p}=0.05)$ indicating a relatively persistent and substantial reduction of energy intake.

In contrast, the model-based calculations demonstrated that energy intake changes followed an exponential time course shown in Figure 1B. Using the clinic weights, $\Delta \mathrm{EI}_{\text {Model }}$ was $-804 \pm 27 \mathrm{kcal} / \mathrm{d}$ averaged over the first 3 months. Over the next 3 months, $\Delta \mathrm{EI}_{\text {Model }}=-279$ $\pm 20 \mathrm{kcal} / \mathrm{d}$ indicating a substantial relaxation of calorie restriction $(\mathrm{p}<0.0001)$ which was again relaxed to $\Delta \mathrm{EI}_{\text {Model }}=-65 \pm 14 \mathrm{kcal} / \mathrm{d}$ between 6 and 12 months $(\mathrm{p}<0.0001)$. In a subset of 307 subjects with self-reported physical activity measurements over the course of the intervention, we found that the $\Delta \mathrm{EI}_{\text {Model }}$ results were within $60 \mathrm{kcal} / \mathrm{d}$ at 3 and 6 months, and within $70 \mathrm{kcal} / \mathrm{d}$ at 12 months, of the corresponding values calculated assuming that physical activity was $10 \mathrm{kcal} / \mathrm{kg} / \mathrm{d}$ throughout the intervention (Supplementary Table 2).

Figures $2 \mathrm{~A}$ and $2 \mathrm{~B}$ show the mean clinic weight changes in the low-carbohydrate and lowfat diet groups, respectively, which were significantly different at 3 months $(\mathrm{p}=0.002)$ and 6 months ( $\mathrm{p}=0.001)$, but not at 12 months $(\mathrm{p}=0.29)$. Weights reported at the group counseling sessions indicated similar degrees of underreporting in each diet group. Self-reported energy intake was not significantly different between low-carbohydrate and low-fat diet groups at 
any time point (Table 1). However, model-based calculations using the clinic weights found that energy intake decreased over the first 3 months by $162 \pm 53 \mathrm{kcal} / \mathrm{d}$ more with the lowcarbohydrate diet group as compared to the low-fat diet $(\mathrm{p}=0.002)$, but there were no significant differences at later times. Figure $2 \mathrm{~B}$ shows that $\Delta \mathrm{EI}_{\text {Model }}$ followed a similar exponential pattern regardless of diet, but the low-carbohydrate diet led to larger early reductions in calorie intake that were not sustained.

Figure 3 depicts individual 12 month clinic weight change data for both the low-fat (left column) and low-carbohydrate (right column) diets as a function of the $\Delta \mathrm{EI}_{\text {Model }}$ calculated using clinic weights averaged over the periods 6-12 months (panel A), 3-6 months (panel B), and 0-3 months (panel C). For the low-fat diet, weight loss at 12 months was correlated with $\Delta \mathrm{EI}_{\text {Model }}$ averaged over 6-12 months ( $\mathrm{r}=0.88$; $\left.\mathrm{p}<0.0001\right), 3-6$ months $(\mathrm{r}=0.79$; $\mathrm{p}<0.0001)$, and $0-3$ months $(\mathrm{r}=0.70 ; \mathrm{p}<0.0001)$. Weight change at 6 months was correlated with $\Delta \mathrm{EI}_{\text {Model }}$ averaged over 3-6 months ( $\left.\mathrm{r}=0.88 ; \mathrm{p}<0.0001\right)$, and $0-3$ months $(\mathrm{r}=0.90$; $\mathrm{p}<0.0001)$ and weight change at 3 months was correlated with $\Delta \mathrm{EI}_{\text {Model }}$ averaged over 0-3 months ( $r=1 ; p<0.0001)$ (not shown). For the low-carbohydrate diet, weight loss at 12 months was correlated with $\Delta \mathrm{EI}_{\text {Model }}$ averaged over 6-12 months ( $\left.\mathrm{r}=0.85 ; \mathrm{p}<0.0001\right), 3-6$ months $(r=0.77 ; p<0.0001)$, and $0-3$ months $(r=0.70 ; p<0.0001)$. Weight change at 6 months was correlated with $\Delta \mathrm{EI}_{\text {Model }}$ averaged over 3-6 months $(\mathrm{r}=0.85 ; \mathrm{p}<0.0001)$, and 0-3 months $(\mathrm{r}=0.87 ; \mathrm{p}<0.0001)$ and weight change at 3 months was correlated with $\Delta \mathrm{EI}_{\text {Model }}$ averaged over $0-3$ months $(r=1 ; p<0.0001)$ (not shown). In contrast, $\Delta \mathrm{EI}_{\text {Recall }}$ was only weakly correlated with contemporaneous weight losses at 3 -months $(\mathrm{r}=0.18 ; \mathrm{p}=0.01)$ and 12-months $(\mathrm{r}=0.18 ; \mathrm{p}=0.01)$ and only for the low-fat diet.

\section{Discussion}

This study demonstrates that the energy intake bias calculated by self-reported $24 \mathrm{hr}$ recall was not constant over time in subjects participating in a low-fat versus low-carbohydrate diet intervention for weight loss. Rather, biases in self-reported energy intake become progressively larger such that early assessments of $\Delta \mathrm{EI}_{\text {Recall }}$ were closer to $\Delta \mathrm{EI}_{\text {Model }}$ as compared to later measurements. Whereas the $\Delta \mathrm{EI}_{\text {Recall }}$ measurements suggested a relatively persistent change in energy intake over time, the calculated average $\Delta \mathrm{EI}_{\text {Model }}$ exhibited a large initial reduction in energy intake that exponentially decayed towards baseline over time. Incorporating self-reported measurements of physical activity throughout the intervention did not materially affect the $\Delta \mathrm{EI}_{\text {Model }}$ results. The low-carbohydrate diet resulted in significantly greater early reductions in model-calculated energy intake, with correspondingly greater early weight losses as compared to the low-fat diet, but these diet differences were not sustained.

The early reductions in $\Delta \mathrm{EI}_{\text {Model }}$ after the onset of the intervention indicated that subjects dramatically cut their calorie intake despite instructions that did not focus on calorie restriction. Rather, the diet instructions emphasized avoiding highly processed foods and reducing dietary carbohydrate or fat to very low levels at the start of the intervention. The model-calculated reductions in energy intake may have been slightly exaggerated at the start of the intervention because they relied on weight losses reported by the subjects at the group counseling sessions which were somewhat greater than could be corroborated at the clinic 
visits. Also, water losses that typically occur at the onset of a weight loss intervention may have amplified the early reductions in energy intake, especially during the initial stages of the low-carbohydrate diet where participants were instructed to reduce digestible carbohydrates to $<20 \mathrm{~g} / \mathrm{d}$ for the first 8 weeks and slowly add back carbohydrates to the minimum sustainable level (3). In this early time period, there was a greater reduction in model-calculated energy intake compared to the low-fat diet which is consistent with greater water losses but may also indicated that very low carbohydrate diets suppress appetite by inducing nutritional ketosis (6). Nevertheless, short-term reductions in appetite did not result in sustained reductions in energy intake with the low-carbohydrate diet and long-term average weight losses were not significantly different between the diets.

The relatively constant self-reported energy intake changes gives the impression that the slowing and plateauing of weight loss was primarily due to reductions in energy expenditure which are known to occur with weight loss (7). However, energy expenditure reductions are quantitatively insufficient to account for the observed body weight trajectory given an approximately constant reduction in energy intake. Thus, energy intake must have risen after its early reduction at the start of the intervention (8). The body weight trajectories observed in the DIETFITS study conform to the ubiquitous slowing of weight loss and subsequent weight plateau after 6-8 months (9) corresponding to exponentially increasing energy intake time course $(10,11)$.

In contrast to the objective measurements of energy intake that exponentially increase over time after the start of the intervention, why do the subjects report a relatively constant reduction in energy intake that progressively deviates from the objective values over time? Perhaps the constant self-reported calorie restriction reflects that the subjects were exerting a persistent effort to adhere to the diet intervention in the face of progressively increasing appetite in proportion to lost weight $(11,12)$. The creeping upwards of actual energy intake over time may have been due to subconscious increases in portion sizes or snacking episodes that failed to register in the repeated 24-hour recalls.

At the end of the 12-month DIETFITS trial, there was a large interindividual variability in weight loss that was associated with the model-calculated energy intake changes at all stages of the intervention. Due to the long time-scale for human body weight to equilibrate to a constant energy intake (8), weight changes over periods of less than a few years are expected to be related to not only current energy intake, but the history of intake changes in the past year or more. Here, we observed that much of the 12-month weight loss variability was associated with energy intake changes occurring in the first few months as well as at later time points. Thus, studies designed to understand weight loss variability need to account for the dynamic nature of human weight loss.

The major limitation of this study was that we did not use doubly labeled water to measure free-living energy intake changes by the gold-standard intake-balance method (13).

However, our mathematical method has been validated against the intake-balance method in a two-year human calorie restriction study (2) that also exhibited a consistent exponential pattern of energy intake changes over time (14). However, this previous validation study did not compare different diets and did not include subjects with obesity (15), so we cannot be 
certain that the model-based calculations of energy intake were valid in the present study population.

In summary, repeated self-reported measurements of energy intake changes during the DIETFITS weight loss intervention were not accurate. Model-based calculations demonstrated an exponential pattern of energy intake change whereby large early calorie reductions decayed back towards baseline over time. Instructions to adhere to a lowcarbohydrate diet resulted in greater calorie restriction compared to a low-fat diet in the early phases of the DIETFITS intervention, but these diet differences were not sustained.

\section{Supplementary Material}

Refer to Web version on PubMed Central for supplementary material.

\section{Acknowledgements}

KDH and JG were supported by the Intramural Research Program of the National Institutes of Health, National Institute of Diabetes and Digestive and Kidney Diseases. JLR and CG were supported by Grant 1R01DK091831 from the NIDDK, Grant 1K12GM088033 from the NIH (CTSA) and the Nutrition Science Initiative. The clinical study protocol and deidentified individual data described in this manuscript will be made available for download on the Open Science Framework website (https://osf.io/) within 6 months of publication for any purpose by anyone.

Funding: KDH and JG were supported by the Intramural Research Program of the National Institutes of Health, National Institute of Diabetes and Digestive and Kidney Diseases. JLR and CG were supported by Grant 1R01DK091831 from the NIDDK, Grant 1K12GM088033 from the NIH (CTSA) and the Nutrition Science Initiative.

ClinicalTrials.gov Identifier: NCT01826591

\section{References}

1. Schoeller DA. How accurate is self-reported dietary energy intake? Nutr Rev 1990;48: 373-379. [PubMed: 2082216]

2. Sanghvi A, Redman LA, Martin CK, Ravussin E, Hall KD. Validation of an inexpensive and accurate mathematical method to measure long-term changes in free-living energy intake. Am J Clin Nutr 2015;102: 353-358. [PubMed: 26040640]

3. Gardner CD, Trepanowski JF, Del Gobbo LC, et al. Effect of low-fat vs low-carbohydrate diet on 12-month weight loss in overweight adults and the association with genotype pattern or insulin secretion: The dietfits randomized clinical trial. JAMA 2018;319: 667-679. [PubMed: 29466592]

4. Stanton MV, Robinson JL, Kirkpatrick SM, Farzinkhou S, Avery EC, Rigdon J, et al. DIETFITS study (diet intervention examining the factors interacting with treatment success) - Study design and methods. Contemporary clinical trials 2017;53: 151-161. [PubMed: 28027950]

5. Sallis JF, Haskell WL, Wood PD, Fortmann SP, Rogers T, Blair SN, et al. Physical activity assessment methodology in the Five-City Project. American journal of epidemiology 1985;121: 91106. [PubMed: 3964995]

6. Gibson AA, Seimon RV, Lee CM, Ayre J, Franklin J, Markovic TP, et al. Do ketogenic diets really suppress appetite? A systematic review and meta-analysis. Obes Rev 2015;16: 64-76. [PubMed: 25402637]

7. Rosenbaum M, Leibel RL. Adaptive thermogenesis in humans. Int J Obes (Lond) 2010;34 Suppl 1: S47-55. [PubMed: 20935667]

8. Hall KD, Sacks G, Chandramohan D, Chow CC, Wang YC, Gortmaker SL, et al. Quantification of the effect of energy imbalance on bodyweight. Lancet 2011;378: 826837. 
9. Franz MJ, VanWormer JJ, Crain AL, Boucher JL, Histon T, Caplan W, et al. Weight-loss outcomes: a systematic review and meta-analysis of weight-loss clinical trials with a minimum 1-year followup. J Am Diet Assoc 2007;107: 1755-1767. [PubMed: 17904936]

10. Gobel B, Sanghvi A, Hall KD. Quantifying energy intake changes during obesity pharmacotherapy. Obesity (Silver Spring) 2014;22: 2105-2108. [PubMed: 24961931]

11. Polidori D, Sanghvi A, Seeley RJ, Hall KD. How Strongly Does Appetite Counter Weight Loss? Quantification of the Feedback Control of Human Energy Intake. Obesity (Silver Spring) 2016;24: 2289-2295. [PubMed: 27804272]

12. Hall KD, Schoeller DA, Brown AW. Reducing Calories to Lose Weight. Jama 2018;319: 23362337.

13. Racette SB, Das SK, Bhapkar M, Hadley EC, Roberts SB, Ravussin E, et al. Approaches for quantifying energy intake and \%calorie restriction during calorie restriction interventions in humans: the multicenter CALERIE study. Am J Physiol Endocrinol Metab 2012;302: E441-448. [PubMed: 22127229]

14. Guo J, Brager DC, Hall KD. Simulating long-term human weight-loss dynamics in response to calorie restriction. Am J Clin Nutr 2018;107: 558-565. [PubMed: 29635495]

15. Ravussin E, Redman LM, Rochon J, Das SK, Fontana L, Kraus WE, et al. A 2-Year Randomized Controlled Trial of Human Caloric Restriction: Feasibility and Effects on Predictors of Health Span and Longevity. J Gerontol A Biol Sci Med Sci 2015;70: 10971104. 


\section{What is already known about this subject?}

- Diet assessments that rely on self-report, such as $24 \mathrm{hr}$ dietary recall, are known to underestimate actual energy intake as measured by doubly labeled water. However, it is possible that repeated self-reported measurements could accurately detect changes in energy intake over time if the absolute bias of self-reported of measurements was approximately constant for each subject.

What this study adds:

- We compared energy intake changes measured using repeated $24 \mathrm{hr}$ dietary recall measurements collected over the course of the 1-year Diet Intervention Examining The Factors Interacting with Treatment Success (DIETFITS) trial versus energy intake changes calculated using repeated body weight measurements as inputs to a validated mathematical model.

- Whereas self-reported measurements indicated a relatively persistent state of calorie restriction, objective model-based measurements demonstrated a large early calorie restriction followed by an exponential rise in energy intake towards the pre-intervention baseline.

- $\quad$ Model-based calculations, but not self-reported measurements, found that low-carbohydrate diets led to significantly greater early decreases in energy intake compared to low-fat diets, but long-term energy intake changes were not significantly different. 

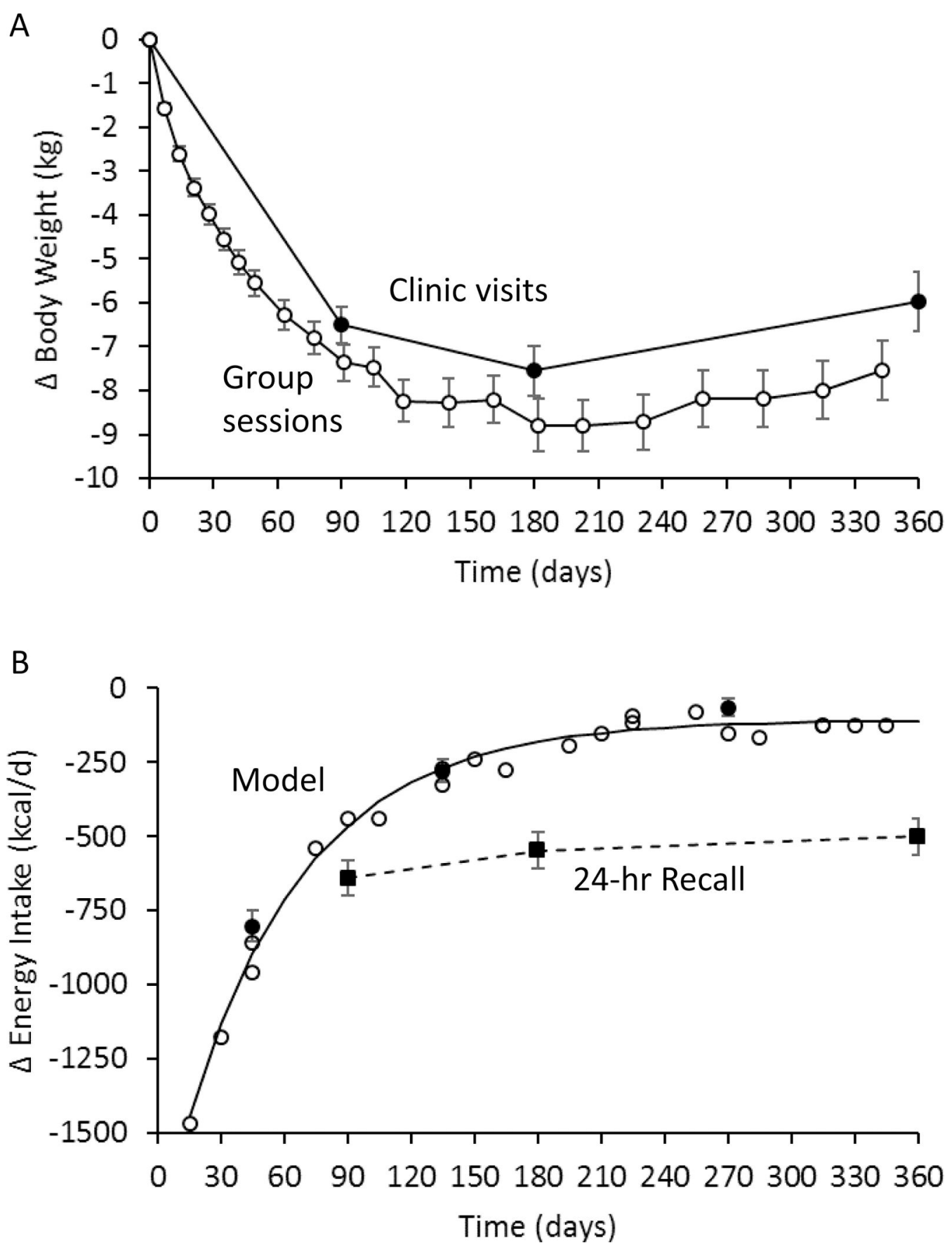

Figure 1.

A) Mean body weight changes measured during the DIETFITS trial clinic visits $(\boldsymbol{O})$ or selfreported by subjects at group counseling sessions $(O)$ for all 414 subjects with complete clinic weight data. B) Mean self-reported energy intake changes ( $\mathbf{\square})$ indicated a relatively persistent reduction in energy intake whereas the model-based measurements ( $\bigcirc$ from selfreported weights and 0 from clinic weights) followed an exponential time course (solid curve). Error bars indicate $95 \%$ CI. 
A

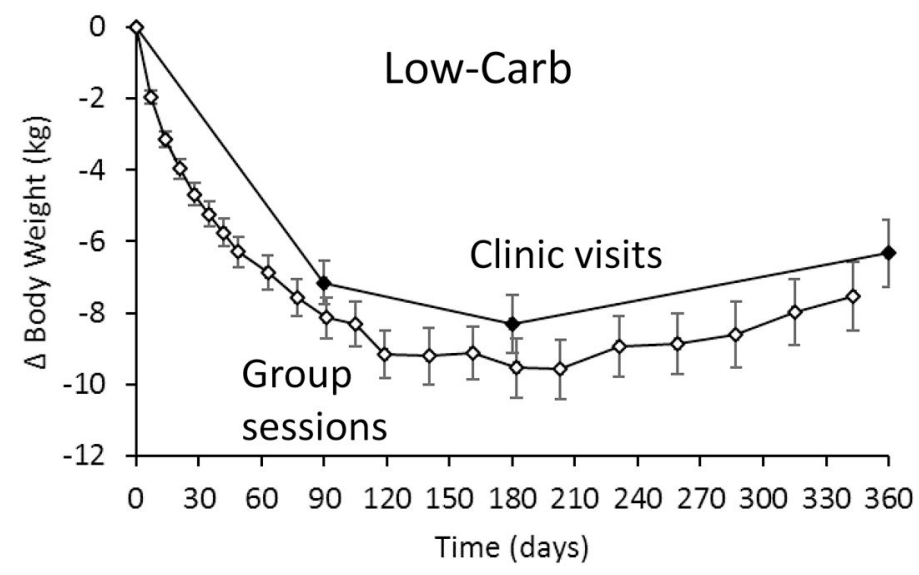

B

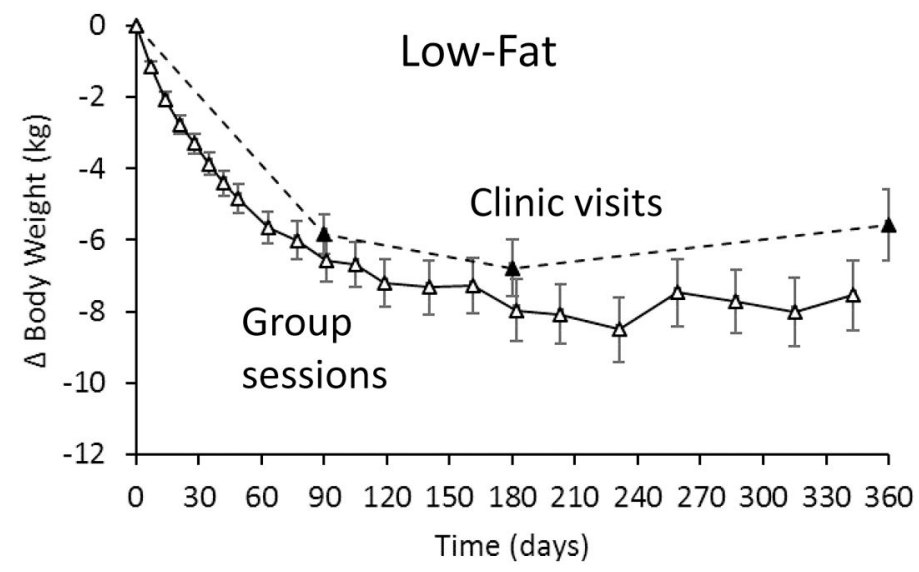

C

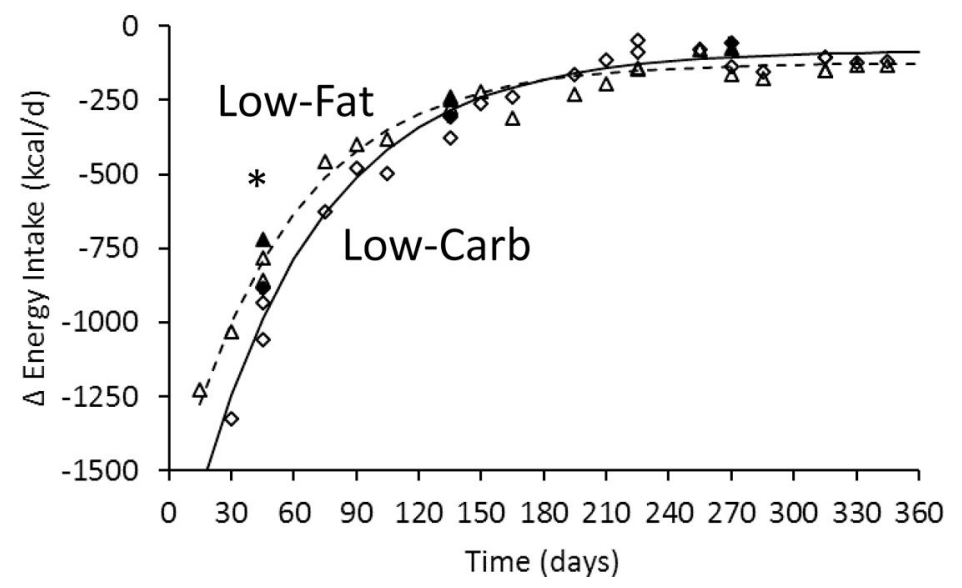

Figure 2.

A) Mean body weight changes for the 209 subjects in the low-carbohydrate diet group ( clinic and $\nabla$ self-reported) and B) the 205 subjects in the low-fat ( $\mathbf{\Delta}$ clinic and $\Delta$ selfreported) diet group. C) Mean model-based measurements of energy intake changes in the low-carbohydrate diet group ( $\boldsymbol{\Delta}$ from clinic weights and $\Delta$ from self-reported weights ) and the low-fat diet group ( $\diamond$ from self-reported weights and from clinic weights) both followed an exponential time courses (solid curve and dashed curve for low-carbohydrate 
and low-fat diets, respectively). * indicates $\mathrm{p}<0.05$ between diet groups and the error bars indicate $95 \%$ CI. 
Low Fat

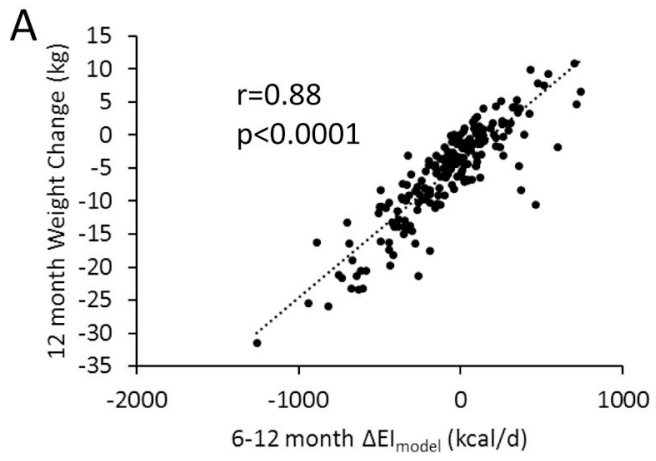

B

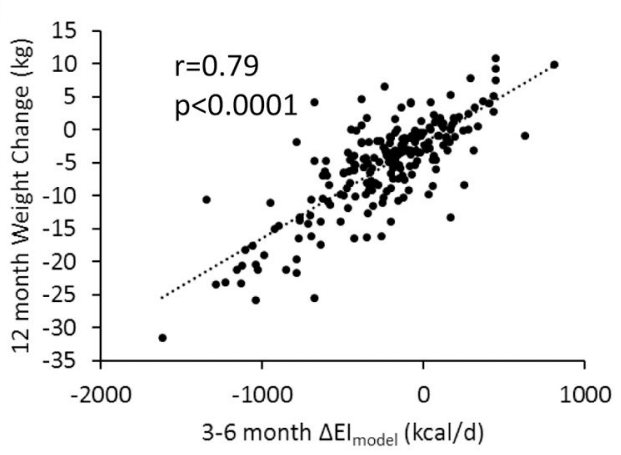

C

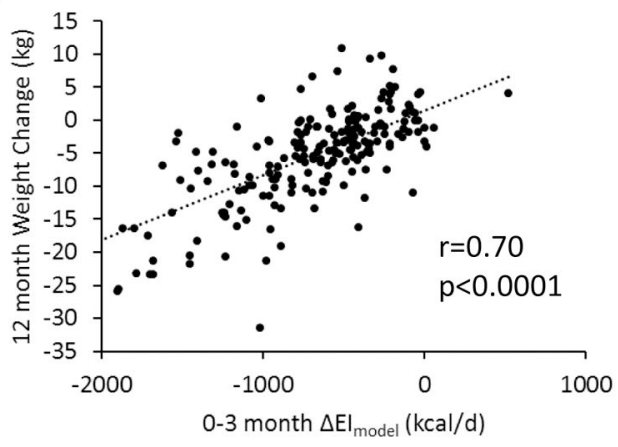

\section{Low Carbohydrate}
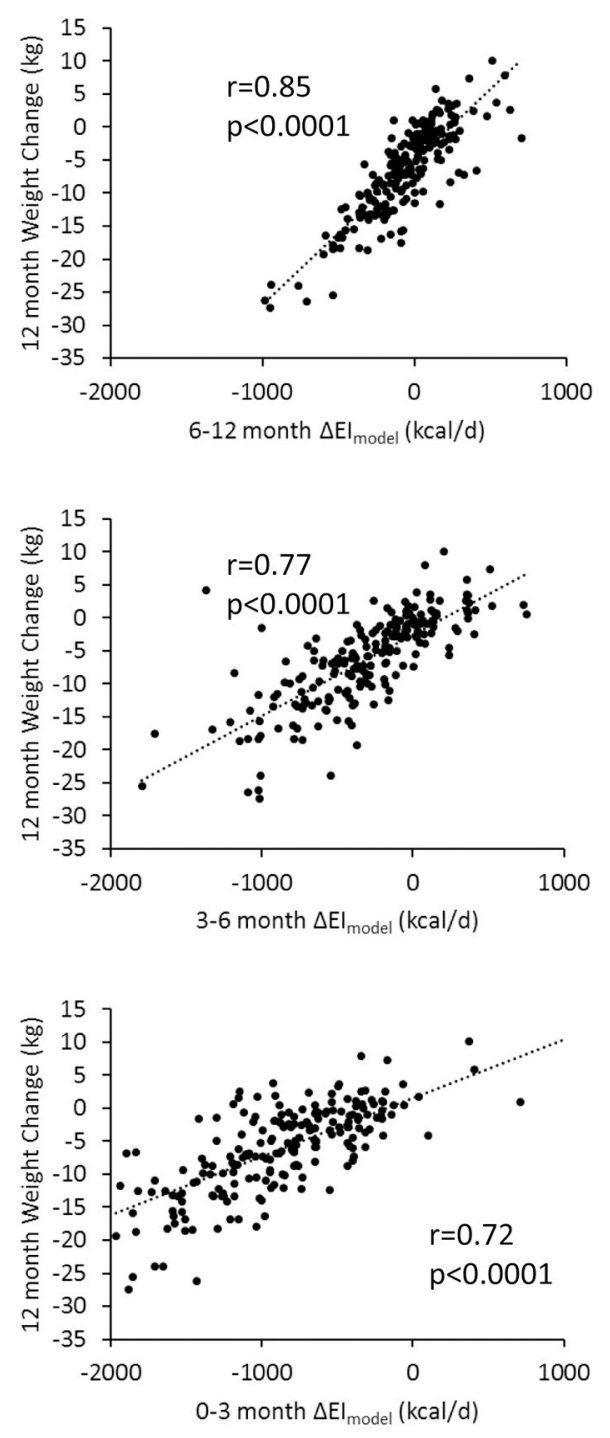

Figure 3.

Individual weight changes at 12 months for subjects assigned to the low-fat diet (left column) and low-carbohydrate diet (right column) were significantly correlated with modelcalculated changes in energy intake averaged over A) 6-12 months; B) 3-6 months; and C) 0-3 months. 
Table 1.

Changes in body weight, self-reported energy intake, and model-claculated energy intake during the DIETFITS intervention (mean $\pm \mathrm{SE}$ ).

\begin{tabular}{|l|c|c|c|c|}
\hline \multicolumn{1}{|c|}{ Variable } & Both Diets $(\mathbf{N}=\mathbf{4 1 4})$ & Low-Carbohydrate $(\mathbf{L C}$; N=209) & Low-Fat $(\mathbf{L F}$; N=205) & P-value LC vs LF \\
\hline$\Delta \mathrm{BW}_{3 \text { months }}$ & $-6.5 \pm 0.2 \mathrm{~kg}$ & $-7.2 \pm 0.3 \mathrm{~kg}$ & $-5.8 \pm 0.3 \mathrm{~kg}$ & 0.002 \\
\hline$\Delta \mathrm{BW}_{6 \text { months }}$ & $-7.6 \pm 0.3 \mathrm{~kg}$ & $-8.3 \pm 0.4 \mathrm{~kg}$ & $-6.8 \pm 0.4 \mathrm{~kg}$ & 0.01 \\
\hline$\Delta \mathrm{BW}_{12 \text { months }} 1$ & $-5.9 \pm 0.3 \mathrm{~kg}$ & $-6.3 \pm 0.5 \mathrm{~kg}$ & $-5.6 \pm 0.5 \mathrm{~kg}$ & 0.29 \\
\hline 3 month $\Delta \mathrm{EI}_{\text {Recall }}$ & $-641 \pm 31 \mathrm{kcal} / \mathrm{d}$ & $-628 \pm 43 \mathrm{kcal} / \mathrm{d}$ & $-653 \pm 44 \mathrm{kcal} / \mathrm{d}$ & 0.68 \\
\hline 6 month $\Delta \mathrm{EI}_{\text {Recall }} 2$ & $-547 \pm 32 \mathrm{kcal} / \mathrm{d}$ & $-552 \pm 45 \mathrm{kcal} / \mathrm{d}$ & $-542 \pm 45 \mathrm{kcal} / \mathrm{d}$ & 0.87 \\
\hline 12 month $\Delta \mathrm{EI}_{\text {Recall }} 3$ & $-500 \pm 31 \mathrm{kcal} / \mathrm{d}$ & $-532 \pm 44 \mathrm{kcal} / \mathrm{d}$ & $-467 \pm 44 \mathrm{kcal} / \mathrm{d}$ & 0.30 \\
\hline $0-3$ month $\Delta \mathrm{EI}_{\text {Model }}$ & $-804 \pm 27 \mathrm{kcal} / \mathrm{d}$ & $-884 \pm 39 \mathrm{kcal} / \mathrm{d}$ & $-722 \pm 36 \mathrm{kcal} / \mathrm{d}$ & 0.002 \\
\hline $3-6$ month $\Delta \mathrm{EI}_{\text {Model }}$ & $-279 \pm 20 \mathrm{kcal} / \mathrm{d}$ & $-307 \pm 29 \mathrm{kcal} / \mathrm{d}$ & $-251 \pm 27 \mathrm{kcal} / \mathrm{d}$ & 0.16 \\
\hline $6-12$ month $\Delta \mathrm{EI}_{\text {Model }}$ & $-65 \pm 28 \mathrm{kcal} / \mathrm{d}$ & $-56 \pm 18 \mathrm{kcal} / \mathrm{d}$ & $-75 \pm 22 \mathrm{kcal} / \mathrm{d}$ & 0.49 \\
\hline
\end{tabular}

1 missing $1 \mathrm{LC}$ value and $2 \mathrm{LF}$ values.

2 missing $4 \mathrm{LC}$ values and $7 \mathrm{LF}$ values.

${ }^{3}$ missing $8 \mathrm{LC}$ values and $5 \mathrm{LF}$ values. 